Published in: Neural Computation 18, 446-469 (2006)

\title{
Magnification Control in Self-Organizing Maps and Neural Gas
}

\author{
Thomas Villmann \\ Clinic for Psychotherapy, University of Leipzig, 04107 Leipzig, Germany \\ Jens Christian Claussen \\ Institute of Theoretical Physics and Astrophysics, \\ Christian-Albrecht University Kiel, 24098 Kiel, Germany
}

We consider different ways to control the magnification in self-organizing maps (SOM) and neural gas (NG). Starting from early approaches of magnification control in vector quantization, we then concentrate on different approaches for SOM and NG. We show that three structurally similar approaches can be applied to both algorithms: localized learning, concave-convex learning, and winner relaxing learning. Thereby, the approach of concave-convex learning in SOM is extended to a more general description, whereas the concave-convex learning for NG is new. In general, the control mechanisms generate only slightly different behavior comparing both neural algorithms. However, we emphasize that the NG results are valid for any data dimension, whereas in the SOM case the results hold only for the one-dimensional case.

\section{Introduction}

Vector quantization is an important task in data processing, pattern recognition and control (Fritzke, 1993; Haykin, 1994; Linde, Buzo, \& Gray, 1980; Ripley, 1996). A large number of different types have been discussed, (for an overview, refer to Haykin, 1994; Kohonen, 1995; Duda \& Hart, 1973). Neural maps are a popular type of neural vector quantizers that are commonly used in, for example, data visualization, feature extraction, principle component analysis, image processing, classification tasks, and acceleration of common vector quantization (de Bodt, Cottrell, Letremy, \& Verleysen, 2004). Well known approaches are the SelfOrganizing Map (SOM) (Kohonen, 1995), the neural gas (NG) (Martinetz, Berkovich, \& Schulten, 1993), elastic net (EN) (Durbin \& Willshaw, 1987) and generative topographic mapping (GTM) (Bishop, Svensén, \& Williams 1998).

In vector quantization, data vectors $\mathbf{v} \in \mathbb{R}^{d}$ are represented by a few codebooks or weight vectors $\mathbf{w}_{i}$, where $i$ is an arbitrary index. Several criteria exist to evaluate the quality of a vector quantizer. The most common 
one is the squared reconstruction error. However, other quality criteria are also known, for instance topographic quality for neighborhood preserving mapping approaches (Bauer \& Pawelzik, 1992; Bauer, Der, \& Villmann, 1999), optimization of mutual information (Linsker, 1989) and other criteria (for an overview, see Haykin, 1994). Generally, a faithful representation of the data space by the codebooks is desired. This property is closely related to the so-called magnification, which describes the relation between data and weight vector density for a given model. The knowledge of magnification of a map is essential for correct interpretation of its output (Hammer \& Villmann, 2003). In addition, explicit magnification control is a desirable property of learning algorithms, if depending on the respective application, only sparsely covered regions of the data space have to be emphasized or, conversely, suppressed. The magnification can be explicitly expressed for several vector quantization models. Usually, for these approaches the magnification can be expressed by a power law between the codebook vector density $\rho$ and the data density $P$. The respective exponent is called magnification exponent or magnification factor. As explained in more detail below, the magnification is also related to other properties of the map, for example, reconstruction error as well as mutual information. Hence, control of magnification is influencing these properties too.

In biologically motivated approaches, magnification can also be seen in the context of information representation in brains, for instance, in the senso-motoric cortex (Ritter, Martinetz, \& Schulten, 1992). Magnification and its control can be related to biological phenomena like the perceptual magnet effect, which refers to the fact that rarely occurring stimuli are differentiated with high precision whereas frequent stimuli are distinguished only in a rough manner (Kuhl, 1991; Kuhl, Williams, Lacerda, Stevens, \& Lindblom, 1992). It is a kind of attention-based learning with inverted magnification, that is, rarely occurring input samples are emphasized by an increased learning gain (Der \& Herrmann, 1992; Herrmann, Bauer, \& Der, 1994). This effect is also beneficial in technical systems. In remotesensing image analysis, for instance, seldomly found ground cover classes should be detected, whereas usual (frequent) classes with broad variance should be suppressed (Merényi \& Jain, 2004; Villmann, Merényi \& Hammer, 2003). Another technical environment for magnification control is robotics for accurate description of dangerous navigation states (Villmann \& Heinze, 2000).

In this article we concentrate on a general framework for magnification control in SOM and NG. In this context, we briefly review the most important approaches. One approach for SOM is generalized, and afterward, it is transferred to NG. For this purpose, we first give the basic notations, followed in section 3 by a more detailed description of magnification and early approaches related to the topic of magnification control, including a unified approach for controlling strategies. The magnification control approaches of SOM are described according to the unified framework in 
section 4 whereby one of them is significantly extended. The same procedure is applied to NG in section 5. Again, one of the control approaches presented in this section is new. A short discussion concludes the article.

\section{Basic Concepts and Notations in SOM and NG}

In general, neural maps project data vectors $\mathbf{v}$ from a (possibly highdimensional) data manifold $\mathcal{D} \subseteq \mathbb{R}^{d}$ onto a set $A$ of neurons $i$, which is formally written as $\Psi_{\mathcal{D} \rightarrow A}: \mathcal{D} \rightarrow A$. Each neuron $i$ is associated with a pointer $\mathbf{w}_{i} \in \mathbb{R}^{d}$, all of which establish the set $\mathbf{W}=\left\{\mathbf{w}_{i}\right\}_{i \in A}$. The mapping description is a winner-take-all rule, that is, a stimulus vector $\mathbf{v} \in \mathcal{D}$ is mapped onto that neuron $s \in A$ with the pointer $\mathbf{w}_{s}$ being closest to the actual presented stimulus vector $\mathbf{v}$,

$$
\Psi_{\mathcal{D} \rightarrow A}: \mathbf{v} \mapsto s(\mathbf{v})=\underset{i \in A}{\operatorname{argmin}}\left\|\mathbf{v}-\mathbf{w}_{i}\right\| .
$$

The neuron $s$ is called winner neuron. The set $R_{i}=$ $\left\{\mathbf{v} \in \mathcal{D} \mid \Psi_{\mathcal{D} \rightarrow A}(\mathbf{v})=i\right\}$ is called the (masked) receptive field of the neuron $i$. The weight vectors are adapted during the learning process such that the data distribution is represented.

For further investigations, we describe SOM and NG as our focused neural maps in more detail. During the adaptation process a sequence of data points $\mathbf{v} \in \mathcal{D}$ is presented to the map with respect to the data distribution $P(\mathcal{D})$. Then the most proximate neuron $s$ according to equation (2.1) is determined, and the pointer $\mathbf{w}_{s}$, as well as all pointers $\mathbf{w}_{i}$ of neurons in the neighborhood of $s$, are shifted towards $\mathbf{v}$, according to

$$
\triangle \mathbf{w}_{i}=\epsilon h(i, \mathbf{v}, \mathbf{W})\left(\mathbf{v}-\mathbf{w}_{i}\right) .
$$

The property of "being in the neighborhood of $s$ " is represented by a neighborhood function $h(i, \mathbf{v}, \mathbf{W})$. The neighborhood function is defined as

$$
h_{\lambda}(i, \mathbf{v}, \mathbf{W})=\exp \left(-\frac{k_{i}(\mathbf{v}, \mathbf{W})}{\lambda}\right)
$$

for the NG, where $k_{i}(\mathbf{v}, \mathbf{W})$ yields the number of pointers $\mathbf{w}_{j}$ for which the relation $\left\|\mathbf{v}-\mathbf{w}_{j}\right\|<\left\|\mathbf{v}-\mathbf{w}_{i}\right\|$ is valid (Martinetz et al., 1993); especially, we have $h_{\lambda}(s, \mathbf{v}, \mathbf{W})=1.0$. In case of SOM the set $A$ of neurons has a topological structure usually chosen as a hypercube or hexagonal lattice. Each neuron $i$ has a fixed position $\mathbf{r}(i)$. The neighborhood function has the form

$$
h_{\sigma}(i, \mathbf{v}, \mathbf{W})=\exp \left(-\frac{\|\mathbf{r}(i)-\mathbf{r}(s(\mathbf{v}))\|_{A}}{2 \sigma^{2}}\right) .
$$


In contrast to the NG, the neighborhood function of SOM is evaluated in the output space $A$ according to its topological structure. This difference causes the significantly different properties of both algorithms. For the SOM there does not exist any energy function such that the adaptation rule follows the gradient descent (Erwin, Obermayer, \& Schulten, 1992). Moreover, the convergence proofs are only valid for the one-dimensional setting (Cottrell, Fort \& Pages, 1998, Ritter et al., 1992). The introduction of an energy function leads to different dynamics as in the EN (Durbin $\&$ Willshaw, 1987) or new winner determination rule (Heskes 1999). The advantage of the SOM is the ordered topological structure of neurons in $A$. In contrast, in the original NG, such an order is not given. One can extend the NG to the topology representing network (TRN) such that topological relations between neurons are installed during learning, although generally they do not achieve the simple structure as in SOM lattices (Martinetz \& Schulten, 1994). Finally, the important advantage of the NG is that the adaptation dynamic of the weight vectors follows a potential minimizing dynamics (Martinetz et al., 1993).

\section{Magnification and Magnification Control in Vector Quantization}

\subsection{Magnification in Vector Quantization.}

Usually vector quantization aims to minimize the reconstruction error

$R E=\sum_{i} \int_{R_{i}}\left\|\mathbf{v}-\mathbf{w}_{i}\right\|^{2} P(\mathbf{v}) d \mathbf{v}$. However, other quality criteria are also known, for instance, topographic quality (Bauer \& Pawelzik, 1992; Bauer et al., 1999). More generally, one can consider the generalized distortion error,

$$
E_{\gamma}=\int_{\mathcal{D}}\left\|\mathbf{w}_{s}-\mathbf{v}\right\|^{\gamma} P(\mathbf{v}) d \mathbf{v} .
$$

This error is closely related to other properties of the (neural) vector quantizer. One important property is the achieved weight vector density $\rho(\mathbf{w})$ after learning in relation to the data density $P(\mathcal{D})$. Generally, for vector quantizers one finds the relation

$$
P(\mathbf{w}) \propto \rho(\mathbf{w})^{\alpha}
$$

after the converged learning process (Zador 1982). The exponent $\alpha$ is called magnification exponent or magnification factor. The magnification is coupled with the generalized distortion error (3.1) by

$$
\alpha=\frac{d}{d+\gamma}
$$


Table 1: Magnification of Different Neural Maps and Vector Quantization Approaches.

\begin{tabular}{lcl}
\hline Model & Magnification & Reference \\
\hline Elastic net & $1+\frac{\kappa}{\sigma^{2}} \frac{1}{P \tilde{\rho}}$ & Claussen and Schuster (2002) \\
SOM & $\frac{1+12 M_{2}(\sigma)}{3+18 M_{2}(\sigma)}$ & Dersch and Tavan (1995) \\
Linsker network & 1 & Linsker (1989) \\
LBG & $\frac{d}{d+2}$ & Zador (1982) \\
FSCL & $\frac{3 \beta+1}{3 \beta+3}$ & Galanopoulos and Ahalt (1996) \\
NG & $\frac{d}{d+2}$ & Martinetz et al. (1993) \\
\hline
\end{tabular}

Note: For SOM, $M_{2}(\sigma)$ denotes the 2nd normalized moment of the neighborhood function depending on the neighborhood range $\sigma$.

where $d$ is the intrinsic or Hausdorff dimension ${ }^{1}$ of the data. Beginning with the pioneering work of Amari (1980), which investigated a resolution-density relation of map formation in a neural field model and extended the approach of Willshaw and von der Malsburg (1976), for several neural map and vector quantizer approaches the magnification relation has been considered, including the investigation of the relation between data and model density.

Generally, different magnification factors are obtained for different vector quantization approaches. An overview of several important models with known magnification factors is given in Table 1

For the usual SOMs, mapping a one-dimensional input space onto a chain of neurons,

$$
\alpha_{S O M}=\frac{2}{3}
$$

holds in the limit $1 \ll \sigma \ll N$ (Ritter \& Schulten, 1986). For small values of neighborhood range $\sigma$, the neighborhood ceases to be of influence, and the magnification rate approaches the value $\alpha=\frac{1}{3}$ (Dersch $\&$ Tavan, 1995). The influence of different types of neighborhood function was studied in detail for SOMs in Dersch and Tavan (1995), which extends the early works of Luttrell (1991) and Ritter (1991). The magnification depends on the second normalized moment $M_{2}$ of the neighborhood function, which itself is determined by the neighborhood range $\sigma$. Van Hulle

\footnotetext{
${ }^{1}$ Several approaches are known to estimate the Hausdorff dimension of data, often called intrinsic dimension. One of the best known methods is the Grassberger-Procacciaanalysis (GP) (Grassberger \& Procaccia, 1983; Takens, 1985). For GP, there is a large number of investigations of statistical properties (e.g., Camastra and Vinciarelli, 2001; Eckmann and Ruelle, 1992; Liebert, 1991; Theiler, 1990). For a neural network approach of intrinsic dimension estimation (based on NG), also in comparison to GP, we refer to Bruske and Sommer (1998), Camastra \& Vinciarelli (2001), Villmann, Hermann and Geyer (2000), Villmann (2002), and Villmann et al. (2003).
} 
(2000) extensively discussed the influence of kernel approaches in SOMs. Results for magnification of discrete SOMs can be found in Ritter (1989) and Kohonen (1999). These latter problems and approaches will not be further addressed here.

According to equations (3.3) and (3.1), the SOM minimizes the somewhat exotic $E_{\frac{1}{2}}$ distortion error, whereas the NG minimizes the usual $E_{2}$ error.

Further, we can observe interesting relations to information-theoretic properties of the mapping: The information transfer realized by the mapping $\Psi_{\mathcal{D} \rightarrow A}$, in general, is not independent of the magnification of the map (Zador, 1982). It has been derived that for an optimal information transfer realizing vector quantizer (or a neural map in our context), the relation $\alpha=1$ holds (Brause, 1992). A vector quantizer designed to achieve an optimal information transfer is the Linsker network (Linsker, 1989; see Table 1), or the optimal coding network approach proposed by Brause (1994).

\subsection{Magnification Control in Vector Quantization: A General Framework.}

As pointed out in section 1 different application tasks may require different magnification properties of the vector quantizer, that is, the magnification should be controlled. Straightforwardly, magnification control means changing the value of the magnification factor $\alpha$ for a given vector quantizer by manipulation of the basic approach.

Consequently, the question is, How one can impact the magnification factor to achieve an a priori chosen magnification factor? We further address this topic in the following. First, we review results from the literature and put them into a general framework.

The first approaches to influence the magnification of a vector quantizer are models of conscience learning, characterized by a modified winner determination. The algorithm by DeSieno (1988) and the frequency sensitive competitive learning (FSCL) (Ahalt, Krishnamurty, Chen, \& Melton, 1990) belong to this algorithm class. Originally, these approaches were proposed for equalizing the winner probability of the neural units in SOM. However, as the neighborhood relation between neurons is not used in this approach, it is applicable to each vector quantizer based on winnertake-all learning. To achieve the announced goal, in the DeSieno model, a bias term $B$ is inserted into the winner determination rule, equation (2.1), such that

$$
\Psi_{\mathcal{D} \rightarrow A}: \mathbf{v} \mapsto s(\mathbf{v})=\underset{i \in A}{\operatorname{argmin}}\left(\left\|\mathbf{v}-\mathbf{w}_{i}\right\|-B\right)
$$

with the bias term $B=\gamma\left(\frac{1}{N}-p_{i}\right)$, and $p_{i}$ is the actual winning probability of the neuron $i$. The algorithm converges such that the winning 
probabilities of all neurons are equalized, which is related to a maximization of the entropy, and, hence, the resulted magnification is equal to the unity. However, an arbitrary magnification can not be achieved. Moreover, as pointed out in van Hulle (2000), the algorithm shows unstable behavior. FSCL modifies the selection criterion for the best-matching unit by a fairness term $F$, which is a function of the winning frequency $\omega_{i}$ of the neurons. Again, the winner determination is modified:

$$
\Psi_{\mathcal{D} \rightarrow A}: \mathbf{v} \mapsto s(\mathbf{v})=\underset{i \in A}{\operatorname{argmin}}\left(F\left(\omega_{i}\right)\left\|\mathbf{v}-\mathbf{w}_{i}\right\|\right) .
$$

As mentioned above, originally it was defined to achieve an equiprobable quantization too. However, it was shown, this goal can not be achieved by the original version (Galanopoulos \& Ahalt, 1996; van Hulle, 2000). Yet for one-dimensional data, any given $\gamma$-norm error criterion, equation (3.1), can be minimized by a specific choice of the fairness function: if $F\left(\omega_{i}\right)$ is taken as

$$
F\left(\omega_{i}\right)=\left(\omega_{i}\right)^{\xi}
$$

for the one-dimensional case a magnification $\alpha_{F S C L}=\frac{3 \beta+1}{3 \beta+3}$ is achieved, being equivalent to $\gamma=\frac{2}{3 \beta+1}$ (Galanopoulos \& Ahalt, 1996). The difficulties of transferring the one-dimensional result to higher dimensions are, however, as prohibitive as in SOM.

We now study control possibilities to achieve arbitrary magnification, focusing on SOM and NG by modification of the learning rule. We emphasize again that for SOM, the results hold only for the one-dimensional case, whereas for NG, the more general case of arbitrary dimensionality is valid. Thus, the following direction of modifications of the general learning rule, equation (2.2),

$$
\triangle \mathbf{w}_{i}=\epsilon h(i, \mathbf{v}, \mathbf{W})\left(\mathbf{v}-\mathbf{w}_{i}\right),
$$

can serve as a general framework:

1. Localized learning: Introduction of a multiplicative factor by a local learning rate $\epsilon_{i}$

2. Winner-relaxing learning: Introduction of winner relaxing by adding a winner-enhancing (relaxing) term $R$

3. Concave-convex learning: Scaling of the learning shift by powers $\xi$ in the factor $\left(\mathbf{v}-\mathbf{w}_{i}\right)^{\xi}$

These three directions serve as axes for a taxonomy in the following section. We focus on SOM and NG as popular neural vector quantizers. We explain, expand and develop the respective methodologies of magnification control for these models. The localized and the winner relaxing learning for SOM and NG are briefly reviewed. In particular, localized learning 
for SOM was published in Bauer, Der, and Herrmann, (1996) whereas winner relaxing learning for both SOM and NG and localized learning in NG were previously developed by the authors (Claussen, 2003, 2005; Claussen \& Villmann, 2003a; Villmann, 2000). The concave-convex learning for SOM is extended here to a more general approach compared to its origins (Zheng \& Greenleaf, 1996). The concave-convex learning for NG is new too.

\section{Controlling the Magnification in SOM}

Within the general framework outlined in section 3.2 we now consider the three learning rule modifications for SOM.

\subsection{Insertion of a Multiplicative Factor: Localized Learning.}

The first choice is to add a factor in the SOM learning rule. An established realization is the localized learning, the biological motivation of which is the perceptual magnet effect (Bauer et al., 1996). For this purpose, an adaptive local learning step size $\epsilon_{s(\mathbf{v})}$ is introduced in equation (2.2) such that the new adaptation rule reads as

$$
\triangle \mathbf{w}_{i}=\epsilon_{s(\mathbf{v})} h_{\sigma}(i, \mathbf{v}, \mathbf{W})\left(\mathbf{v}-\mathbf{w}_{i}\right)
$$

where $s(\mathbf{v})$ is being the best-matching neuron with respect to equation (2.1). The local learning rates $\epsilon_{i}=\epsilon\left(\mathbf{w}_{i}\right)$ depend on the stimulus density $P$ at the position of their weight vectors $\mathbf{w}_{i}$ via

$$
\left\langle\epsilon_{i}\right\rangle=\epsilon_{0} P\left(\mathbf{w}_{i}\right)^{m}
$$

where the brackets $\langle\ldots\rangle$ denote the average in time. This approach leads to the new magnification law,

$$
\alpha_{\text {localSOM }}^{\prime}=\alpha_{S O M} \cdot(m+1)
$$

where $m$ appears to be an explicit control parameter (Bauer et al., 1996). Hence, an arbitrary predefined magnification can be achieved.

In applications, one has to estimate the generally unknown data distribution $P$, which may lead to numerical instabilities of the control mechanism (van Hulle, 2000).

\subsection{Winner-Relaxing SOM and Magnification Control.}

Recently, a new approach for magnification control of the SOM by a generalization (Claussen, 2003, 2005) of the winner-relaxing modification (Kohonen, 1991) was derived, giving a control scheme, which is independent 
of the shape of the data distribution (Claussen 2005). We refer to this algorithm as WRSOM.

In the original winner-relaxing SOM, an additional term occurs in learning for the winning neuron only, implementing a relaxing behavior. The relaxing force is a weighted sum of the difference between the weight vectors and the input according to their neighborhood relation. The relaxing term was introduced to obtain a learning dynamic for SOM according to an average reconstruction error taking into account the effect of shifting Voronoi borders.

The original learning rule is added by a winner relaxing term $R(\mu, \kappa)$ as

$$
\triangle \mathbf{w}_{i}=\epsilon h_{\sigma}(i, \mathbf{v}, \mathbf{W})\left(\mathbf{v}-\mathbf{w}_{i}\right)+R(\mu, \kappa),
$$

with $R(\mu, \kappa)$ being

$$
\begin{aligned}
R(\mu, \kappa)= & (\mu+\kappa)\left(\mathbf{v}-\mathbf{w}_{i}\right) \delta_{i s} \\
& -\kappa \delta_{i s} \sum_{j} h_{\sigma}(j, \mathbf{v}, \mathbf{W})\left(\mathbf{v}-\mathbf{w}_{j}\right),
\end{aligned}
$$

depending on weighting parameters $\mu$ and $\kappa$. For $\mu=0$ and $\kappa=\frac{1}{2}$, the original winner relaxing SOM is obtained (Kohonen, 1991). Surprisingly, it has been shown that the magnification is independent of $\mu$ (Claussen, $2003,2005)$. Only the choice of $\kappa$ contributes to the magnification:

$$
\alpha_{W R S O M}^{\prime}=\frac{2}{\kappa+3} .
$$

The stability range is $|\kappa| \leq 1$, which restricts the accessible magnification range to $\frac{1}{2} \leq \alpha_{W R S O M}^{\prime} \leq 1$. More detailed numerical simulations and stability analysis can be found in Claussen (2005).

The advantage of winner relaxing learning is that no estimate of the generally unknown data distribution has to be made, as required in the local learning approach above.

\subsection{Concave-Convex Learning.}

The third structural possibility for control according to our framework is to apply concave or convex learning in the learning rule. This approach was introduced in Zheng and Greenleaf (1996). Here, we extend this approach to a more general variant.

Originally, an exponent $\xi$ is introduced in the general learning rule such that equation (2.2) now reads as

$$
\triangle \mathbf{w}_{i}=\epsilon h_{\sigma}(i, \mathbf{v}, \mathbf{W})\left(\mathbf{v}-\mathbf{w}_{i}\right)^{\xi}
$$


with

$$
\left(\mathbf{v}-\mathbf{w}_{i}\right)^{\xi \stackrel{\text { def }}{=}}\left(\mathbf{v}-\mathbf{w}_{i}\right) \cdot\left\|\mathbf{v}-\mathbf{w}_{i}\right\|^{\xi-1}
$$

Thereby, two different possibilities are proposed: $\xi=\frac{1}{\kappa}$ with $\kappa>1, \kappa \in \mathbb{N}$ and $\kappa$ is odd (convex learning), or one simply takes $\xi>1, \xi \in \mathbb{N}$ and $\xi$ is odd (concave learning). This gives the magnification

$$
\begin{aligned}
\alpha_{\text {concave/convex } S O M}^{\prime} & =\frac{2}{\xi+2} \\
& =\alpha_{S O M} \cdot \frac{3}{\xi+2}
\end{aligned}
$$

which allows an explicit magnification control. Yet this approach allows only a rather rough control around $\xi=1$ : the neighboring allowed values are $\xi=\frac{1}{3}$ and $\xi=3$ corresponding to magnifications $\alpha_{\text {concave } / \text { convex } S O M}^{\prime}=$ $\frac{6}{7}$ and $\alpha_{\text {concave } / \text { convexSOM }}^{\prime}=\frac{2}{5}$, respectively. Therefore, greater flexibility would be of interest.

For this purpose, we are seeking for a generalization of both concave and convex learning. As a more general choice we take $\xi$ to be real, that is, $\xi \in \mathbb{R}$. If we do so, the same magnification equation (4.9) is obtained. The proof of the magnification law is given in appendix A. Obviously, the choices $\xi=\frac{1}{\kappa}$ and $\xi=\kappa>1, \kappa \in \mathbb{N}$ and $\kappa$ being odd as made in Zheng and Greenleaf (1996) are special cases of the now general approach.

We considered the numerical behaviour of the magnification control of the WRSOM using a one-dimensinal chain of 50 neurons. The data distribution was chosen in agreement with Bauer et al. (1996) as $P(x)=$ $\sin (\pi x)$. The theoretical entropy maximum of the winning probabilities of the neurons $p_{i}$ is $\sum_{i=1}^{N} p_{i} \log \left(p_{i}\right)=\log (N)$ giving the value 3.912 for $N=50$. The results in dependence on $\xi$ for different neighborhood ranges $\sigma$ are depicted in Figure 1 .

According to the theoretical prediction, the output entropy is maximized for small $\xi$, and for large $\xi$, an magnification exponent zero is reached corresponding to an equidistant codebook without adaptation to the input distribution. For $\sigma<1$, the turnover is shifted toward smaller values of $\xi$, and for $\xi \ll 1, \sigma \ll 1$, fluctuations increase.

Further, as in the WRSOM, the advantage of concave-convex learning is that no estimate of the generally unknown data distribution has to be made as before in localized learning. 


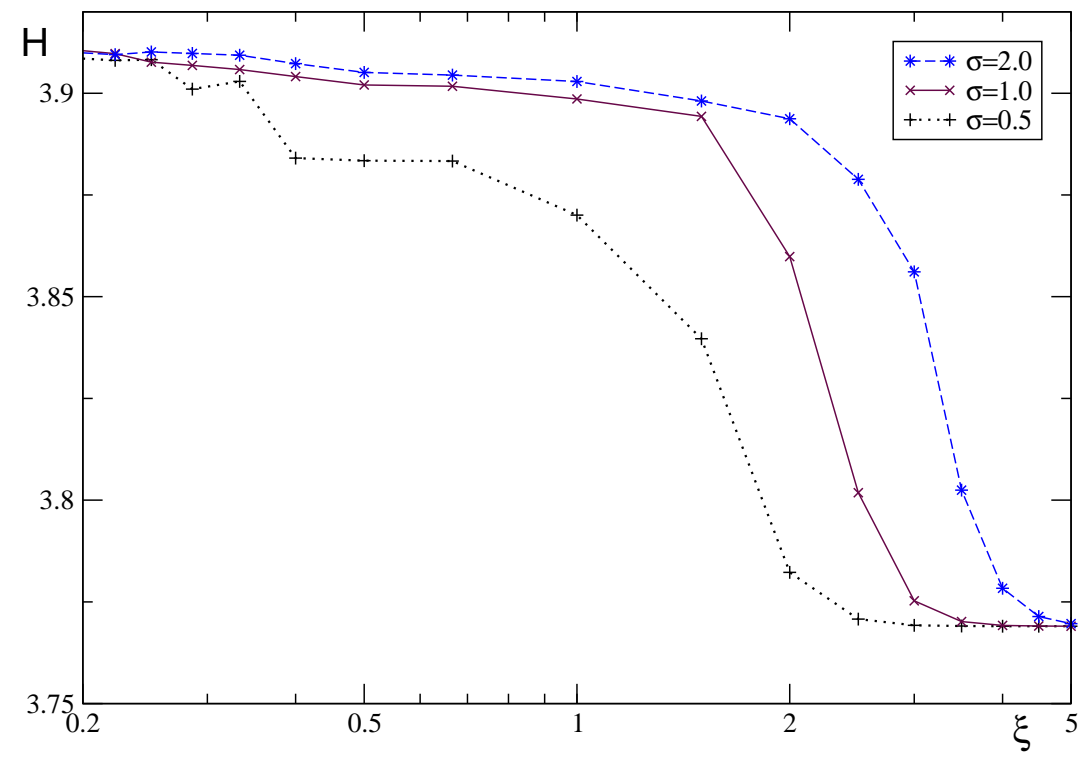

Figure 1: Output entropy for concave and convex learning. An input density of $P(x)=\sin (\pi x)$ was presented to an one-dimensional chain of $N=50$ neurons after $10^{6}$ learning steps of stochastic sequential updating, averaged over $10^{5}$ inputs, and learning rate $\epsilon=0.01$, fixed.

\section{Magnification Control in Neural Gas}

In this section we transfer the ideas of magnification control in SOM to the NG, keeping in mind the advantage that the results then are valid for any dimension.

\subsection{Multiplicative Factor - Localized Learning.}

The idea of localized learning is now applied to NG (Herrmann \& Villmann 1997). Hence, we have the localized learning rule

$$
\triangle \mathbf{w}_{i}=\epsilon_{s(\mathbf{v})} h_{\lambda}(i, \mathbf{v}, \mathbf{W})\left(\mathbf{v}-\mathbf{w}_{i}\right),
$$

with $s(\mathbf{v})$ again being the best-matching neuron with respect to equation (2.1) and $\epsilon_{s(\mathbf{v})}$ is the local learning chosen as in equation (4.2). This approach gives a similar result as for SOM,

$$
\alpha_{\text {local } N G}^{\prime}=\alpha_{N G} \cdot(m+1),
$$




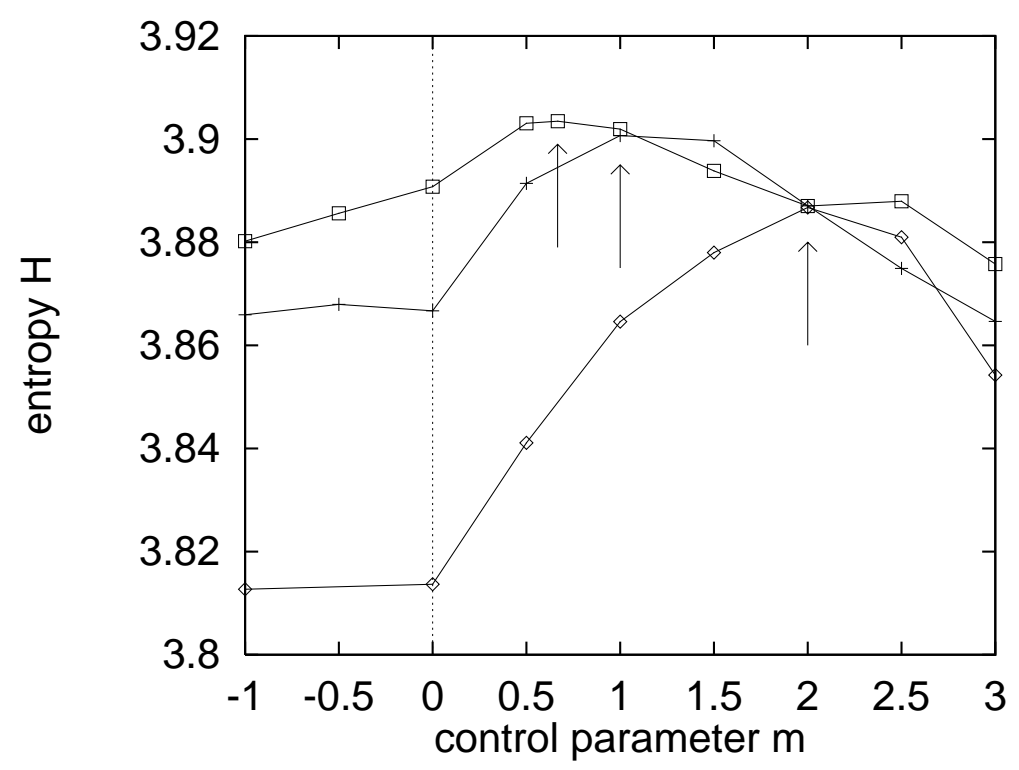

Figure 2: Local learning for NG: Plot of the entropy $H$ for maps trained with different magnification control parameters $m(d=1(\diamond), d=2(+), d=3(\square))$. The arrows indicate the theoretical values of $m(m=2, m=1, m=2 / 3$, resp.) which maximizes the entropy of the map.

and, hence, allows a magnification control (Villmann, 2000). However, we have similar restrictions as for SOM: in actual applications one has to estimate the generally unknown data distribution $P$.

The numerical study shows that the approach can also be used to increase the mutual information of a map generated by a NG (Villmann, 2000). As for WRSOM, we use a standard setup as in Villmann (2000) of 50 Neurons and $10^{7}$ training steps with a probability density $P\left(x_{1} \ldots x_{d}\right)=\prod_{i} \sin \left(\pi x_{i}\right), x \in[0,1]$, and with parameters $\lambda=1.5$ fixed and $\epsilon$ decaying from 0.5 to 0.05 . The entropy of the resulting map computed for an input dimension of 1, 2 and 3 is plotted in Figure 2

\subsection{Winner-Relaxing NG.}

The winner-relaxing NG (WRNG) was first studied in Claussen and Villmann (2003a). According to the WRSOM approach, one uses an additive winner relaxing term $R(\mu, \kappa)$ to the original learning rule:

$$
\triangle \mathbf{w}_{i}=\epsilon h_{\lambda}(i, \mathbf{v}, \mathbf{W})\left(\mathbf{v}-\mathbf{w}_{i}\right)+R(\mu, \kappa),
$$


with $R(\mu, \kappa)$ being as in equation (4.5). The resulting WRNGmagnification for small neighborhood values $\lambda$ with $\lambda \rightarrow 0$ but not vanishing is given by Claussen and Villmann (2005):

$$
\alpha_{W R N G}^{\prime}=\frac{1}{1-\kappa} \frac{d}{d+2}
$$

Thereby, the magnification exponent appears to be independent of an additional diagonal term (controlled by $\mu$ ) for the winner the same as in WRSOM; again $\mu=0$ is the usual setting. If the same stability borders $|\kappa|=1$ of the WRSOM also apply here, one can expect to increase the NG exponent by positive values of $\kappa$, or to lower the NG exponent by factor $1 / 2$ for $\kappa=-1$.

However, one has to be cautious when transferring the $\lambda \rightarrow 0$ result obtained above (which would require to increase the number of neurons as well) to a realistic situation where a decrease of $\lambda$ with time will be limited to a final finite value to avoid the stability problems found in Herrmann and Villmann (1997). For a finite $\lambda$ the maximal coefficient $h_{\lambda}$ that contributes to the averaged learning shift is given by the prefactor of the second but one winner, which is given by $\mathrm{e}^{\lambda}$ (Claussen $\&$ Villmann, 2005). For the $\mathrm{NG}$, however, the neighborhood is defined by the rank list. As the winner term of the NG is not present in the winner relaxing term (for $\mu=0$ ), all terms share the factor $\mathrm{e}^{-\lambda}$ by $h_{\lambda}(k)=\mathrm{e}^{-\lambda} h_{\lambda}(k-1)$ which indicates that in the discretized algorithm $\kappa$ has to be rescaled by $\mathrm{e}^{+\lambda}$ to agree with the continuum theory. The numerical investigation indicates that this prefactor applies for finite $\lambda$ and number of neurons. The scaling of the position of the entropy maximum with input dimension is in agreement with theory, as well as the prediction of the opposite sign of $\kappa$ that has to be taken to increase mutual information.

Numerical studies show that winner-relaxing learning can also be used to increase the mutual information of a NG vector quantization. The entropy shows a dimension-dependent maximum approximately at $\kappa=$ $\frac{2}{d+2} \mathrm{e}^{\lambda}$ (see Figure 3). In any case, within a broad range around the optimal $\kappa$, the entropy is close to the maximum.

The advantage of the method is to be independent on estimation of the unknown data distribution as the SOM equivalent WRSOM. Further, again as in the WRSOM, the magnification of WRNG is independent in the first order on the diagonal term, controlled by $\mu$. Numerical simulations have shown that the contribution in higher orders is marginal (Claussen \& Villmann, 2003b). More pronounced is the influence of the diagonal term on stability. According to the larger prefactor, no stable behavior has been found for $|\mu| \geq 1$, therefore $\mu=0$ is the recommended setting (Claussen \&Villmann, 2005). 


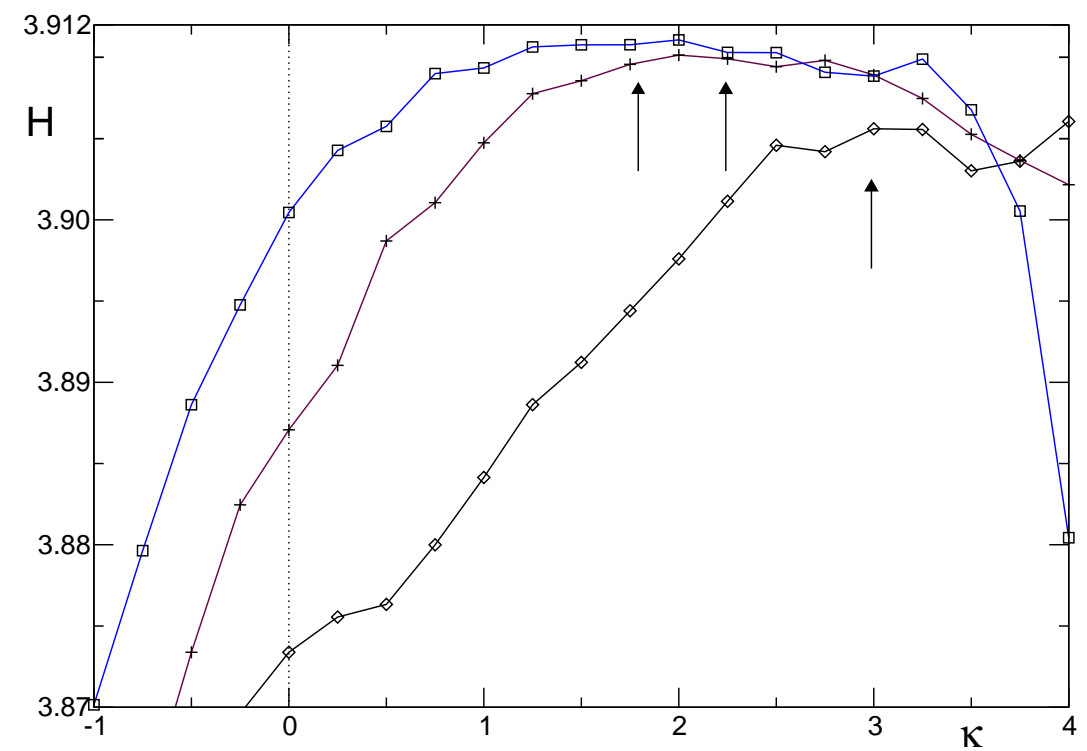

Figure 3: Winner relaxing learning for NG: Plot of the entropy $H$ curves for varying values of $\kappa$ for one-, two and three-dimensional data. The entropy has the maximum if the magnification equals the unit (Zador 1982). The arrows indicate the $\kappa$-values for the respective data dimensions.

\subsection{Concave-Convex Learning.}

We now consider the third modification known from SOM, the concaveconvex learning approach but in its new, developed general variant,

$$
\triangle \mathbf{w}_{i}=\epsilon h_{\lambda}(i, \mathbf{v}, \mathbf{W})\left(\mathbf{v}-\mathbf{w}_{i}\right)^{\xi},
$$

with $\xi \in \mathbb{R}$ and the definition (4.8). It is proved in the appendix B that the resulting magnification is

$$
\alpha_{\text {concave/convex } N G}^{\prime}=\frac{d}{\xi+1+d},
$$

depending on the intrinsic data dimensionality $d$. This dependency is in agreement with the usual magnification law of NG, which is also related to the data dimension.

The respective numerical simulations with the parameter choice as before are given in Figure 4. In contrast to concave-convex SOM where $\alpha^{\prime}=1$ can be achieved for large $\xi$, here $\alpha^{\prime}$ is bounded by $\frac{d}{d+1}$; information optimal learning is not possible in cases of low-dimensional data. 


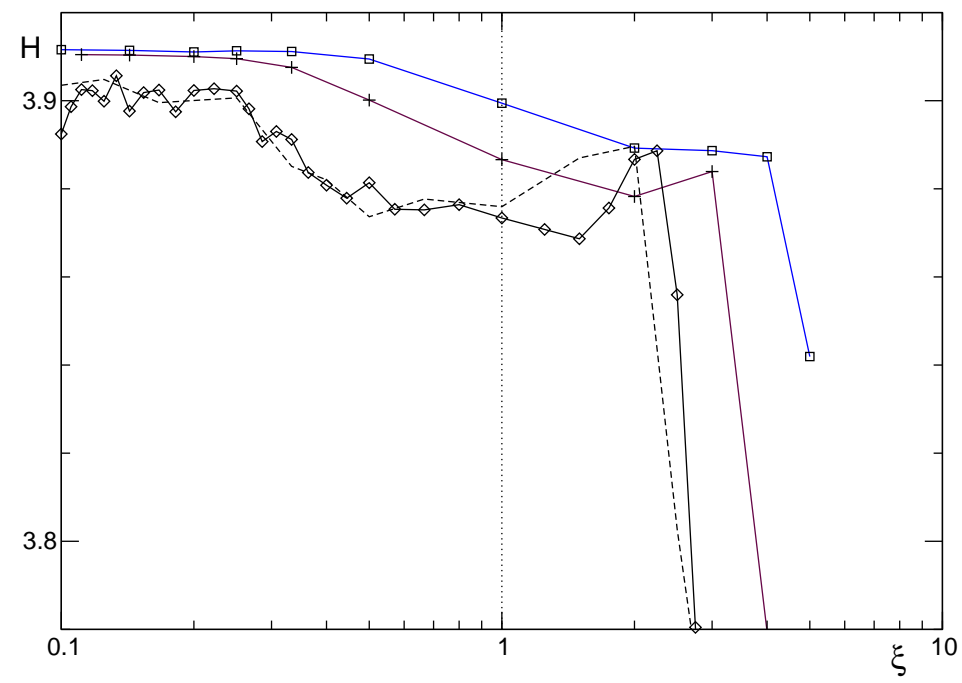

Figure 4: Concave-convex learning for NG: Plot of the entropy $H$ curves for varying values of $\xi$ for one-, two and three-dimensional data. The entropy can be enhanced by convex learning in each case (dashed line: $d=1$, with $10^{8}$ learning steps).

\section{Discussion}

According to the given general framework, we studied three structurally different approaches for magnification control in SOM and NG. All methods are capable to control the magnification with more or less accuracy. Yet, they differ in properties (e.g., stability range, density estimation). No approach yet shows a clear advantage. The choice of the optimal algorithm may depend on the particular problem and implementation constraints. In particular, several problems occur in actual applications. First, in the SOM case, all result are only valid for the one-dimensional case, because all investigations are based on the usual convergence dynamic. However, the SOM dynamics is analytically treatable only in the one-dimensional setting and higher-dimensional cases that factorize. Moving away from these special cases causes a systematic shift in magnification control, as numerically shown in Jain and Merényi (2004). In actual applications, a quantitative comparison with theory is quite limited due to several influences which are not easily tractable. First, the data density has to be estimated, which is generally difficult (Merényi \& Jain, 2004); second, the intrinsic dimension has to be determined; and third, the measurement of the magnification from the density of weight vectors is rather coarse, especially in higher dimensions. 
Table 2: Comparison of Magnification Control for the Different Control Approaches for SOM and NG $(d=1$ for SOM).

\begin{tabular}{lcc}
\hline & SOM & NG \\
\hline $\begin{array}{l}\text { Local } \\
\text { learning }\end{array}$ & $(m+1) \alpha_{S O M}$ & $(m+1) \alpha_{N G}$ \\
$\begin{array}{l}\text { Winner-relaxing } \\
\text { learning }\end{array}$ & $\frac{3}{\kappa+3} \alpha_{S O M}$ & $($ Villmann 2000) \\
Concave-convex & $\frac{1}{1-\kappa} \alpha_{N G}$ \\
learning & $\frac{3}{\xi+2} \alpha_{S O M}$ & (Claussen et al., 1996) \\
& (in section 4.3; & $\frac{d+2}{d+\xi+1} \alpha_{N G}$ \\
& Zheng \& Greenleaf, 1996) & \\
\hline
\end{tabular}

Only some special cases can be handled adequately. In particular, maximizing mutual information can be controlled easily by observation of the entropy of winning probabilities of neurons or consideration of inverted magnification in case of available auxiliary class information, that is, labeled data (Merényi \& Jain, 2004). Thus, actual applications have to be done carefully using some heuristics. Interesting, successful applications of magnification control (by local learning) in satellite remote sensing image analysis can be found in Merényi and Jain (2004), (Villmann, 1999; Villmann et al., 2003).

Summarizing the above approaches of magnification control, we obtain the good news that the possibilities for magnification control known from SOM can be successfully transferred to the NG learning in all three cases. The achieved theoretical magnifications are collected in Table 2 .

The interesting point is that the local learning approach, as well as concave-convex learning, yields structurally similar modification factors for the new magnification. However, a magnification of 1 is not reachable by concave-convex learning in case of NG. In case of the winner relaxing approach, we have a remarkable difference: in contrast to the WRSOM, where the relaxing term has to be inverted $(\kappa<0)$ to increase the magnification exponent, for the $\mathrm{NG}$, positive values of $\kappa$ are required to increase the magnification factor.

\section{Appendix A: Magnification Law of the Generalized Concave-Convex Learning for the Self-Organizing Map}

In this appendix we prove the magnification law of the generalized concave-convex learning for SOM: the exponent in equation (4.7) is required to be $\xi \in \mathbb{R}$ and keeping further in mind the definition (4.8). Since the convergence proofs of SOM are only valid for the one-dimensional setting, we switch from $\mathbf{w}$ to $w$ and from $\mathbf{v}$ to $v$. 
In the continuum approach, we can replace the index of the neuron by its position or location $r$ (Ritter et al., 1992). Further, the neighborhood function $h_{\sigma}$ depends only on the difference of the location $r$ to $r_{s(v)}$ as the location of the winning neuron. Then we have in the equilibrium for the learning rule equation (4.7),

$$
\int h_{\sigma}\left(r-r_{s(v)}\right)(v-w(r))^{\xi} P(v) d v=0 .
$$

We perform the usual approach of expanding the integrand in a Taylor series in powers of $\varsigma=s(v)-r$ and evaluating at $r$ (Ritter \& Schulten, 1986; Hertz, Krogh, \& Palmer, 1991; Zheng \& Greenleaf, 1996). This gives

$$
v=w(r+\varsigma)
$$

$h_{\sigma}(s(v)-r)$ becomes $h_{\sigma}(\varsigma)=h_{\sigma}(-\varsigma)$, and

$$
P(v)=P(w(r+\varsigma)) \approx P(w)+\varsigma P^{\prime}(w) w^{\prime}(r) .
$$

Further, $d v=d w(r+\varsigma)=w^{\prime}(r+\varsigma) d \varsigma$ can be rewritten as

$$
w^{\prime}(r+\varsigma) d \varsigma \approx\left(w^{\prime}+\varsigma w^{\prime \prime}\right) d \varsigma,
$$

and for $v-w(r)=w(r+\varsigma)-w(r)$ we get

$$
w(r+\varsigma)-w(r) \approx \varsigma w^{\prime}+\frac{1}{2} \varsigma^{2} w^{\prime \prime}=\varsigma\left(w^{\prime}+\frac{1}{2} \varsigma w^{\prime \prime}\right) .
$$

Because of $(v-w(r))^{\xi}$ in equation A.1, we consider $\left(w^{\prime}+\frac{1}{2} \varsigma w^{\prime \prime}\right)^{\xi}$ :

$$
\left(w^{\prime}+\frac{1}{2} \varsigma w^{\prime \prime}\right)^{\xi} \approx\left(w^{\prime}\right)^{\xi}\left(1+\frac{\varsigma \cdot \xi}{2} \frac{w^{\prime \prime}}{w^{\prime}}\right) .
$$

Further, because of the definition (4.8), the power $\varsigma^{\xi}$ has to be interpreted as

$$
\varsigma^{\xi}=\varsigma \cdot|\varsigma|^{\xi-1}
$$

which is an odd function in $\varsigma$.

Collecting now A.2 A.7 we get in A.1

$$
\begin{aligned}
0= & \int h_{\sigma}(\varsigma) \cdot \varsigma \cdot|\varsigma|^{\xi-1} \cdot\left(w^{\prime}\right)^{\xi} \cdot\left(1+\frac{1}{2} \xi w^{\prime \prime}\left(w^{\prime}\right)^{-1} \varsigma\right) \\
& \times\left(P(w)+\varsigma P^{\prime}(w) w^{\prime}(r)\right)\left(w^{\prime}+\varsigma w^{\prime \prime}\right) d \varsigma .
\end{aligned}
$$


Since $\varsigma \cdot|\varsigma|^{\xi-1}$ is odd, the term of lowest order in $\varsigma$ vanishes according to the rotational symmetry of $h_{\sigma}(\varsigma)$. Further, in our approximation, we ignore terms behind $\varsigma^{2}$. Hence, the above equation can be simplified as

$$
0=\left(w^{\prime}\right)^{\xi}\left(P^{\prime}(w)\left(w^{\prime}\right)^{2}+\frac{\xi+2}{2} P(w) w^{\prime \prime}\right) \int h_{\sigma}(\varsigma) \cdot \varsigma^{2} \cdot|\varsigma|^{\xi-1} d \varsigma
$$

From there we get

$$
\rho=\left|\frac{d r}{d w}\right|=P^{\frac{2}{2+\xi}}
$$

and, hence,

$$
\alpha_{\text {concave/convexSOM }}=\frac{2}{2+\xi},
$$

which completes the proof.

\section{Appendix B: Magnification Law of the Generalized Concave-Convex Learning for Neural Gas}

For the derivation of the magnification for the generalized concave-convex learning in case of magnification-controlled NG, first we have the usual continuum assumption (Ritter et al., 1992). The further treatment is in complete analogy to the derivation of the magnification in the usual NG (Martinetz et al., 1993). Let $\mathbf{r}$ be the difference vector

$$
\mathbf{r}=\mathbf{v}-\mathbf{w}_{i}
$$

The winning rank $k_{i}(\mathbf{v}, \mathbf{W})$ in the neighborhood function $h_{\lambda}(i, \mathbf{v}, \mathbf{W})$ in equation (2.3) depends only on $\mathbf{r}$, therefore, we introduce the new variable

$$
\mathbf{x}(\mathbf{r})=\hat{\mathbf{r}} \cdot \mathbf{k}_{i}(\mathbf{r})^{\frac{1}{d}}
$$

which can be assumed as monotonously increasing with $\|\mathbf{r}\|$. We define the $d \times d$-Jacobian

$$
\mathbf{J}(\mathbf{x})=\operatorname{det}\left(\frac{\partial r_{k}}{\partial x_{l}}\right)
$$


Starting from the new learning rule,

$$
\triangle \mathbf{w}_{i}=\epsilon h_{\lambda}(i, \mathbf{v}, \mathbf{W})\left(\mathbf{v}-\mathbf{w}_{i}\right)^{\xi},
$$

again consider the averaged change,

$$
\left\langle\triangle \mathbf{w}_{i}\right\rangle=\int P(\mathbf{v}) h_{\lambda}(i, \mathbf{v}, \mathbf{W})\left(\mathbf{v}-\mathbf{w}_{i}\right)^{\xi} d \mathbf{v}
$$

If $h_{\lambda}(i, \mathbf{v}, \mathbf{W})$ in equation (2.3) rapidly decreases to zero with increasing $\mathbf{r}$, we can replace the quantities $\mathbf{r}(\mathbf{x}), \mathbf{J}(\mathbf{x})$ by the first terms of their respective Taylor expansions around the point $\mathbf{x}=0$, neglecting higher derivatives. We obtain

$$
\mathbf{x}(\mathbf{r})=\mathbf{r}\left(\tau_{d} \rho\left(\mathbf{w}_{i}\right)\right)^{\frac{1}{d}}\left(1+\frac{\mathbf{r} \cdot \partial_{\mathbf{r}} \rho\left(\mathbf{w}_{i}\right)}{d \cdot \rho\left(\mathbf{w}_{i}\right)}+\mathcal{O}\left(\mathbf{r}^{2}\right)\right)
$$

which corresponds to

$$
\mathbf{r}(\mathbf{x})=\frac{\left(1-\left(\tau_{d} \rho\left(\mathbf{w}_{i}\right)\right)^{-\frac{1}{d}} \cdot \frac{\mathbf{x} \cdot \partial_{\mathbf{r}} \rho\left(\mathbf{w}_{i}\right)}{d \cdot \rho\left(\mathbf{w}_{i}\right)}+\mathcal{O}\left(\mathbf{x}^{2}\right)\right)}{\mathbf{x}\left(\tau_{d} \rho\left(\mathbf{w}_{i}\right)\right)^{\frac{1}{d}}}
$$

with

$$
\tau_{d}=\frac{\pi^{\frac{d}{2}}}{\Gamma\left(\frac{d}{2}+1\right)}
$$

as the volume of a $d$-dimensional unit sphere (Martinetz et al., 1993). We define $\varphi=\tau_{d} \rho\left(\mathbf{w}_{i}\right)$. Further, we expand $\mathbf{J}(\mathbf{x})$ and obtain

$$
\begin{aligned}
\mathbf{J}(\mathbf{x}) & =\left(\mathbf{J}(0)+x_{k} \frac{\partial \mathbf{J}}{\partial x_{k}}+\ldots\right) \\
& =\frac{1}{\varphi}\left(1-\varphi^{-\frac{1}{d}}\left(1+\frac{1}{d}\right) \cdot \mathbf{x} \cdot \frac{\partial_{\mathbf{r}} \rho}{\rho}\right)+\mathcal{O}\left(x^{2}\right)
\end{aligned}
$$

and, hence,

$$
\left.\frac{\partial \mathbf{J}}{\partial \mathbf{x}}\right|_{\mathbf{x}=0}=-\varphi^{-\left(1+\frac{1}{d}\right)} \frac{\partial_{\mathbf{r}} \rho}{\rho}
$$


After collecting all replacements, (B.5) becomes

$$
\begin{aligned}
\left\langle\triangle \mathbf{w}_{i}\right\rangle= & \epsilon \cdot \varphi^{-\frac{\xi}{d}} \int_{\mathcal{D}} d \mathbf{x} h_{\lambda}(\mathbf{x}) \cdot \mathbf{x}^{\xi} \\
& \cdot\left(P+\varphi^{-\frac{1}{d}} \cdot \mathbf{x} \cdot \partial_{\mathbf{r}} P+\ldots\right) \\
& \cdot\left(\frac{1}{\varphi}-\left(1+\frac{1}{d}\right) \varphi^{-\left(1+\frac{1}{d}\right)} \cdot \mathbf{x} \cdot \frac{\partial_{\mathbf{r}} \rho}{\rho}+\ldots\right) \\
& \cdot\left(1-\varphi^{-\frac{1}{d}} \cdot \mathbf{x} \cdot \frac{\partial_{\mathbf{r}} \rho}{d \cdot \rho}+\ldots\right)^{\xi}
\end{aligned}
$$

with new integration variable $\mathbf{x}$. We use the approximation

$$
\left(1-\varphi^{-\frac{1}{d}} \cdot \mathbf{x} \cdot \frac{\partial_{\mathbf{r}} \rho}{d \cdot \rho}+\ldots\right)^{\xi} \approx 1-\xi \varphi^{-\frac{1}{d}} \cdot \mathbf{x} \cdot \frac{\partial_{\mathbf{r}} \rho}{d \cdot \rho}+\ldots
$$

and get

$$
\begin{aligned}
\left\langle\triangle \mathbf{w}_{i}\right\rangle= & \epsilon \cdot \varphi^{-\frac{\xi}{d}} \int_{\mathcal{D}} d \mathbf{x} h_{\lambda}(\mathbf{x}) \cdot \mathbf{x}^{\xi} \\
& \cdot\left(P+\varphi^{-\frac{1}{d}} \cdot \mathbf{x}^{\xi} \cdot \partial_{\mathbf{r}} P+\ldots\right) \\
& \cdot\left(\frac{1}{\varphi}-\left(1+\frac{1}{d}\right) \varphi^{-\left(1+\frac{1}{d}\right)} \cdot \mathbf{x}^{\xi} \cdot \frac{\partial_{\mathbf{r}} \rho}{\rho}+\ldots\right) \\
& \cdot\left(1-\xi \varphi^{-\frac{1}{d}} \cdot \mathbf{x}^{\xi} \cdot \frac{\partial_{\mathbf{r}} \rho}{d \cdot \rho}+\ldots\right) .
\end{aligned}
$$

In the equilibrium $\left\langle\triangle \mathbf{w}_{i}\right\rangle=0$, we have

$$
\begin{aligned}
0= & \int_{\mathcal{D}} d \mathbf{x} h_{\lambda}(\mathbf{x}) \cdot \mathbf{x}^{\xi} \cdot\left(P+\varphi^{-\frac{1}{d}} \cdot \mathbf{x}^{\xi} \cdot \partial_{\mathbf{r}} P+\ldots\right) \\
& \cdot\left(\frac{1}{\varphi}-\left(1+\frac{1}{d}\right) \varphi^{-\left(1+\frac{1}{d}\right)} \cdot \mathbf{x}^{\xi} \cdot \frac{\partial_{\mathbf{r}} \rho}{\rho}+\ldots\right) \\
& \cdot\left(1-\xi \varphi^{-\frac{1}{d}} \cdot \mathbf{x}^{\xi} \cdot \frac{\partial_{\mathbf{r}} \rho}{d \cdot \rho}+\ldots\right) .
\end{aligned}
$$

Because of the rotational symmetry of $h_{\lambda}$, we can neglect odd power terms in $\mathbf{x}$. Remaining terms are of even power order. Again, according to equation (4.8), we take $\mathbf{x}^{\xi}=\mathbf{x} \cdot|\mathbf{x}|^{\xi-1}$, and, hence, $\mathbf{x}^{\xi}$ itself acts as an odd term. 
Therefore, only terms containing $\mathbf{x}^{\xi+k}$ with odd $k$ contribute. Finally, considering the non-vanishing terms and neglecting higher order terms, we find the relation

$$
\frac{\partial_{\mathbf{r}} P}{P\left(\mathbf{w}_{i}\right)}=\frac{\partial_{\mathbf{r}} \rho}{\rho}\left(\frac{d}{d+\xi+1}\right),
$$

which is the desired result.

\section{Acknowledgements}

We are grateful to Barbara Hammer (University Clausthal, Germany) for intensive discussions and comments. We also thank an anonymeous reviewer for a hint that led us to a more elegant proof in appendix A.

\section{References}

Ahalt, S. C., Krishnamurty, A. K. Chen, P., \& Melton, D. E. (1990). Competitive learning algorithms for vector quantization. Neural Networks, 3, 277-290.

Amari, S.-I. (1980). Topographic organization of nerve fields. Bulletin of Mathematical Biology, 42, 339-364.

Bauer, H. U., Der, R., \& Herrmann M. (1996). Controlling the magnification factor of self-organizing feature maps. Neural Computation, 8, 757-771.

Bauer, H. U., Der, R., \& Villmann, Th. (1999). Neural maps and topographic vector quantization. Neural Networks, 12, 659-676.

Bauer, H. U., \& Pawelzik, K. R. (1992). Quantifying the neighborhood preservation of Self-Organizing Feature Maps. IEEE Trans. on Neural Networks, 3, 570-579.

Bishop, C. M., Svensén, M., \& Williams, C. K. I. (1998). GTM: The generative topographic mapping. Neural Computation, 10, 215-234.

Brause, R. (1992). Optimal information distribution and performance in neighbourhood-conserving maps for robot control. Int. J. Computers and Artificial Intelligence, 11, 173-199.

Brause, R. W. (1994). An approximation network with maximal transinformation. In Maria Marinaro and Pietro G. Morasso, editors, Proc. ICANN'94, International Conference on Artificial Neural Networks, vol. I, pp. 701-704, Springer, London.

Bruske, J., \& Sommer, G. (1998). Intrinsic dimensionality estimation with optimally topology preserving maps. IEEE Transactions on Pattern Analysis and Machine Intelligence, 20, 572-575.

Camastra, F. \& Vinciarelli, A. (2001). Intrinsic dimension estimation of data: an approach based Grassberger-Procaccia's algorithm. Neural Processing Letters, $14,27-34$.

Claussen J. C. (2003). Winner-relaxing and winner-enhancing Kohonen maps: Maximal mutual information from enhancing the winner. Complexity, 8(4), $15-22$.

Claussen J. C. (2005). Winner-Relaxing Self-Organizing Maps. Neural Computation, 17, 997-1009. 
Claussen J. C. \& H. G. Schuster, H. G. (2002). Asymptotic level density of the Elastic Net self-organizing feature map. In J.R. Dorronsoro, editor, Proc. International Conf. on Artificial Neural Networks (ICANN), Lecture Notes in Computer Science 2415, pages 939-944. Springer Verlag.

Claussen J. C. \& Villmann, Th. (2003a). Magnification control in winner relaxing neural gas. In M. Verleysen, editor, Proc. Of European Symposium on Artificial Neural Networks(ESANN'2003), pages 93-98, d-side, Brussels, Belgium.

Claussen J. C. \& Villmann, Th. (2003b). Magnification control in neural gas by winner relaxing learning: Independence of a diagonal term. In Kaynak, O., Alpaydin, E., Oja, E., and Xu, L. editors, Proc. International Conference on Artificial Neural Networks (ICANN/ICONIP 2003), pages 58-61, Bogazici University, Istanbul.

Claussen J. C. \& Villmann, Th. (2005). Magnification control in winner-relaxing neural gas. Neurocomputing, 63, 125-137.

Cottrell, M., Fort, J. C., \& Pages, G. (1998). Theoretical aspects of the SOM algorithm. Neurocomputing, 21, 119-138.

de Bodt, E., Cottrell, M., Letremy, P., \& Verleysen, M. (2004). On the use of self-orgainzing maps to accelerate vector quantization. Neurocomputing, 17, 187-203.

Der, R., \& Herrmann, M. (1992). Attention based partitioning. In M. Van der Meer, editor, Bericht des Status-Seminar des BMFT Neuroinformatik, pages 441-446. DLR (Berlin).

Dersch, D., \& Tavan., P. (1995). Asymptotic level density in topological feature maps. IEEE Trans. on Neural Networks, 6, 230-236.

DeSieno, D. (1988). Adding a conscience to competitive learning. In Proc. ICNN'88, International Conference on Neural Networks, pages 117-124, Piscataway, NJ, 1988. IEEE Service Center.

Duda, R. O., \& Hart, P. E. (1973). Pattern Classification and Scene Analysis. Wiley, New York, 1973.

Durbin, R. \& D. Willshaw, D. (1987) An analogue approach to the travelling salesman problem using an elastic net method. Nature, 326, 689-691.

Eckmann, J. P., \& Ruelle, D. (1992). Fundamental limitations for estimating dimensions and Lyapunov exponents in dynamical systems. Physica D, 56, 185-187.

Erwin, E., Obermayer, K., \& Schulten, K. (1992). Self-organizing maps: Ordering, convergence properties and energy functions. Biol. Cyb., 67, 47-55.

Fritzke, B. (1993). Vector quantization with a growing and splitting elastic net. In Stan Gielen and Bert Kappen, editors, Proc. ICANN'93, International Conference on Artificial Neural Networks, pages 580-585, Springer, London, UK.

Galanopoulos, A. S., \& Ahalt, S. C. (1996). Codeword distribution for frequency sensitive competitive learning with one dimensional input data. IEEE Transactions on Neural Networks, 7, 752-756.

Grassberger, P. and Procaccia, I. (1983). Measuring the strangeness of strange attractors. Physica D, 9, 189-208.

Hammer, B., \& Villmann, Th. (2003). Mathematical aspects of neural networks. In M. Verleysen, editor, Proc. Of European Symposium on Artificial Neural Networks (ESANN'2003), pages 59-72, d-side, Brussels, Belgium.

Haykin, S. (1994). Neural Networks - A Comprehensive Foundation. IEEE Press, New York.

Herrmann, M., Bauer, H.-U., \& Der, R. (1994). The 'perceptual magnet' effect: A model based on self-organizing feature maps. In L. S. Smith and P. J. B. Hancock, editors, Neural Computation and Psychology, pages 107-116, Stirling, Springer-Verlag. 
Herrmann, M., \& Villmann, Th. (1997). Vector quantization by optimal neural gas. In W. Gerstner, A. Germond, M. Hasler, \& J.-D. Nicoud (eds.), Artificial Neural Networks - Proceedings of International Conference on Artificial Neural Networks (ICANN'97) Lausanne, pages 625-630. Lecture Notes in Computer Science 1327, Springer Verlag, Berlin Heidelberg.

Hertz, J. A., Krogh, A., \& Palmer, R. G. (1991). Introduction to the Theory of Neural Computation, volume 1 of Santa Fe Institute Studies in the Sciences of Complexity: Lecture Notes. Addison-Wesley, Redwood City, CA.

Heskes, T. (1999). Energy functions for self-organizing maps. In E. Oja and S. Kaski, editors, Kohonen Maps, pages 303-316. Elsevier, Amsterdam.

Jain, A. \& Merényi, E. (2004). Forbidden magnification? I. In M. Verleysen, editor, European Symposium on Artificial Neural Networks 2004, pages 51-56. d-side publications.

Kohonen, T. (1991). Self-Organizing Maps: Optimization approaches. In T. Kohonen, K. Mäkisara, O. Simula, and J. Kangas, editors, Artificial Neural Networks, volume II, pages 981-990, Amsterdam, Netherlands, 1991. North-Holland.

Kohonen, T. (1995). Self-Organizing Maps, volume 30 of Springer Series in Information Sciences. Springer, Berlin, Heidelberg. (Second Extended Edition 1997).

Kohonen, T. (1999). Comparison of SOM point densities based on different criteria. Neural Computation, 11, 2081-95.

Kuhl, P. K. (1991). Human adults and human infants show a 'perceptual magnet' effect for the prototypes of speech categories, monkeys do not. Perception and Psychophysics, 50, 93-107.

Kuhl, P. K., Williams, K. A., Lacerda, F., Stevens, K. N. \& Lindblom, B. (1992). Linguistic experience alters phonetic perception in infants by 6 months of age. Science, 255, 606-608.

Liebert, W. (1991). Chaos und Herzdynamik. Verlag Harri Deutsch, Frankfurt/M., Germany.

Linde, Y., Buzo, A., \& Gray, R. M. (1980). An algorithm for vector quantizer design. IEEE Transactions on Communications, 28, 84-95.

Linsker, R. (1989). How to generate maps by maximizing the mutual information between input and output signals. Neural Computation, 1, 402-411.

Luttrell, S. P. (1991). Code vector density in topographic mappings: scalar case. IEEE Trans. on Neural Networks, 2, 427-436.

Martinetz, Th. M., Berkovich, S. G., \& Schulten, K. J. (1993). "Neural-gas" network for vector quantization and its application to time-series prediction. IEEE Trans. on Neural Networks, 4, 558-569.

Martinetz, Th., \& Schulten, K. (1994). Topology representing networks. Neural Networks, 7, 507-522.

Merényi, E., \& Jain, A. (2004). Forbidden magnification? II. In M. Verleysen, editor, European Symposium on Artificial Neural Networks 2004, pages 57-62. d-side publications.

Ripley, B. D. (1996). Pattern Recognition and Neural Networks. Cambridge University Press.

Ritter, H. (1989). Asymptotic level density for a class of vector quantization processes. Report A9, Helsinki University of Technology, Laboratory of Computer and Information Science, Espoo, Finland.

Ritter, H. (1991). Asymptotic level density for a class of vector quantization processes. IEEE Trans. on Neural Networks, 2, 173-175.

Ritter, H., \& Schulten, K. (1986). On the stationary state of Kohonen's self-organizing sensory mapping. Biol. Cyb., 54, 99-106. 
Ritter, H., Martinetz, Th., \& Schulten, K. (1992). Neural Computation and SelfOrganizing Maps: An Introduction. Addison-Wesley, Reading, MA.

Takens, F. (1985). On the numerical determination of the dimension of an attractor. In B. Braaksma, H. Broer, and F. Takens, editors, Dynamical Systems and Bifurcations, pages 99-106, Springer, Berlin.

Theiler, J. (1990). Satistical precision of dimension estimators. Physical Review A, 41, 3038-3051.

van Hulle, M. M. (2000). Faithful Representations and Topographic Maps From Distortion- to Information-based Self-organization. J. Wiley \& Sons, Inc.

Villmann, Th. (1999). Benefits and limits of the self-organizing map and its variants in the area of satellite remote sensoring processing. In Proc. Of European Symposium on Artificial Neural Networks (ESANN'99), pages 111-116, D facto publications, Brussels, Belgium.

Villmann, Th. (2000). Controlling strategies for the magnification factor in the neural gas network. Neural Network World, 10, 739-750.

Villmann, Th. (2002). Neural maps for faithful data modelling in medicine - state of the art and exemplary applications. Neurocomputing, 48, 229-250.

Villmann, Th., \& Heinze, A. (2000). Application of magnification control for the neural gas network in a sensorimotor architecture for robot navigation. In HorstMichael Groß, Klaus Debes, and Hans-Joachim Böhme, editors, Proceedings of Selbstorganisation Von Adaptivem Verfahren (SOAVE'2000) Ilmenau, pages 125-134, VDI-Verlag Düsseldorf, 2000. Fortschrittsberichte des VDI.

Villmann, Th., Hermann, W., \& Geyer, M. (2000). Variants of self-organizing maps for data mining and data visualization in medicine. Neural Network World, 10, 751-762.

Villmann, Th., Merényi, E., \& Hammer, B. (2003). Neural maps in remote sensing image analysis. Neural Networks, 16, 389-403.

Willshaw, D. J., \& von der Malsburg, C. (1976). How patterned neural connections can be set up by self-organization. Proceedings of the Royal Society of London, Series B, 194, 431-445.

Zador, P. L. (1982). Asymptotic quantization error of continuous signals and the quantization dimension. IEEE Transaction on Information Theory, 28,149-159.

Zheng, Y., \& Greenleaf, J. F. (1996). The effect of concave and convex weight adjustments on self-organizing maps. IEEE Transactions on Neural Networks, 7, 87-96.

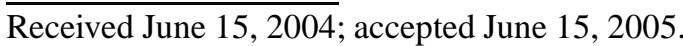

